\title{
Multi-Agent Personal Memory Assistant
}

\author{
Ângelo Costa ${ }^{1}$, Paulo Novais ${ }^{2}$, Ricardo Costa ${ }^{3}$, Juan M. Corchado ${ }^{4}$ and José \\ Neves $^{2}$ \\ ${ }^{1,2}$ CCTC, Departamento de Informática, Universidade do Minho, Braga, Portugal \\ ${ }^{3}$ CIICESI, College of Management and Technology of Polytechnic of Porto, Felgueiras, \\ Portugal \\ ${ }^{4}$ Departamento Informática y Automática, University of Salamanca, Salamanca, Spain \\ 1 angelogoncalocosta@gmail.com, ${ }^{2}$ \{pjon,jneves\}@di.uminho.pt, \\ ${ }^{3}$ rcosta@estgf.ipp.pt, ${ }^{4}$ corchado@usal.es
}

\begin{abstract}
Memory is one of our most precious goods has it gives us the ability to store, retain and recall information thus giving a meaning to our past and help us to envision our future, dreams and expectations. However, ageing decreases the capacity of remembering and the capacity to store new memories, thus affecting our life quality. These presented problems configure a social and human dilemma. With the presented work we intend to address some of these problems, thru the use of the Personal Memory Assistant (PMA) concept in order to help its user to remember things and occurrences in a proactive manner. We will also address socialization and relaxation events that should be part of the user's life. With the use of a Multi-Agent System to implement the PMA, the objectives can be achieved in a ubiquitous and highly configurable manner. It is presented here the platform concept, scheme and the agent characteristics and their contribution to each and every agent.
\end{abstract}

Keywords: Multi-agent systems, e-Health, Memory Assistant, Ambient Assisted Living, Scheduling

\section{Introduction}

An intrinsic characteristic of the human being is his memory. Our remembering capacity has the utmost importance as it gives us the sense of being and the capacity to have a social life. It makes objects to have a meaning and it keeps the instructions of how things are done. This work is oriented to an older population, typically retired, helping them in scheduling events and suggest activities, in order to fill their unfilled time, with minimal interaction. To achieve this we have created a platform based on a Multi-Agent System that can, through embedded intelligent and recurring to other collaborative agents, address it. On top of this platform a Personal Memory Assistant and a Social Enabler where developed and are here presented. We are also going to demonstrates that a distributed system approach is adequate for developing multi-agent systems for healthcare provision tools, helping improving the life of its users, focusing on the PMA paradigm. 


\section{Ageing Factors}

The life expectancy of the world population, according to the United Nations Population Fund (UNPF), is increasing and the birth rate of children is decreasing rapidly. The UNPF estimates that in a period of fifty years the European Population decreased $13 \%$, increasing the age average to 48 years old [1]. At the age of 50 the human beings begin to be affected by it, being the forgetfulness of more re-cent events, one of the most occurred symptoms.

In most cases the loss of memory is more likely to appear and will keep advancing in parallel with ageing and there is little to do in terms of regaining the memorizing capabilities, the most it can be done is exercising the brain through specific brain training exercises [2]. There are three main stages of loss of memory: Cognitively Unimpaired, Mild Cognitive Impairment and Severe Cognitive Impairment (Dementia). Each stage requires different attention and focus suited to their conditionings. Additionally we should also consider the possible memory problems associated with diseases, especially chronic ones, that affects our ability to remember, meaning we can have younger people affected with this problem.

There is still no known way of reversing the human brain loss of information, so a possible solution may be the use of computational systems to store and retrieve all that data. As studies have been done in the area of human interaction and wellbeing, it is know that scheduling and storing, intelligently, user's activities makes communication easier with their peers and relatives, thus greatly improve the elderly self-esteem on their daily activities [3] [4].

\section{3 iGenda: Scheduling and Organization}

The main objective in this work is to present an intelligent scheduler that interacts with its users through computational means, creating a product that will help remember relevant information and events, a PMA [5]. This is done by trying to emulating the way the brain processes new events and reorganizes already scheduled ones. To reschedule an event we normally see in the agenda which are the events able to be moved to another place, considering factors like their relevance and possible problems (e.g. other persons involved in a specific event). It is specially aimed to help the ones with loss of memory, by sustaining all the daily events and warning the user when it is time to put them into action. It will be able to receive information delivered by any source and organise it in the most convenient way, according to predefined standards and protocols, so that the user will not need to manually plan or schedule specific events and tasks. The iGenda is a hierarchy of Agents (Fig. 1) that follow specific tasks and protocols [6], intended to deal with its user's expectations. The main agents are the Agenda Manager (AM), the Free Time Manager (FTM), the Conflicts Manager (CM) and the Interface Manager (IM). All the project agents were constructed to function on the JADE platform, having the decision making processes been written in Logic 
Programming Language (LPL), namely Prolog in order to the results to be assertive and logically correct.

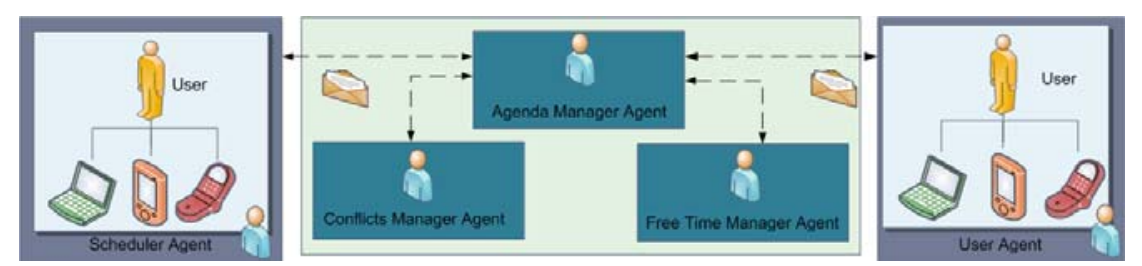

Fig. 1. Modules Scheme of iGenda.

We approached the problem by constructing a Multi-Agent System [6] that is able to support Communication and Decision Making Agents, each one with unique characteristics in a non-competitive and parallel functioning environment so agents can separately process different calendars and events, making different choices. They obviously cannot write to the same calendar at the same time so they are also non-cancelling each other. Also, with the utilization of JADE, it is possible to distribute agent platforms across several machines, which may have different operative systems, with the possibility to migrate them during run-time. It also provides portability, which means that any module may run in different machines, being positioned on any part of the world.

\section{Agents Communication}

The proposed environment is a distributed one, so it is essential to provide the necessary mechanisms for cooperation and coordination in order to allow the agents to proceed, correctly, with their tasks. The communication between the several existing agents can be established by several means of communication like Ethernet, WIFI, GSM, UMTS and others (Fig. 2).

The communication protocol complies with the FIPA (Foundation for Intelligent Physical Agents) standard ACL, being the JADE platform used to build the iGenda architecture (Fig. 3). All the agents are compliant with this standard since they are all JADE implemented agents. Messages carry the information of updates and direct announcements. These messages are sent and received through the several modules and clients. There are already some agents being developed in JADE-Leap [7] for the needed interaction with mobile devices and also an early implementation and structuring of the project in JADEX [8] as it is our belief that the BDI will help to design and implement future agents that will be added to the system. 


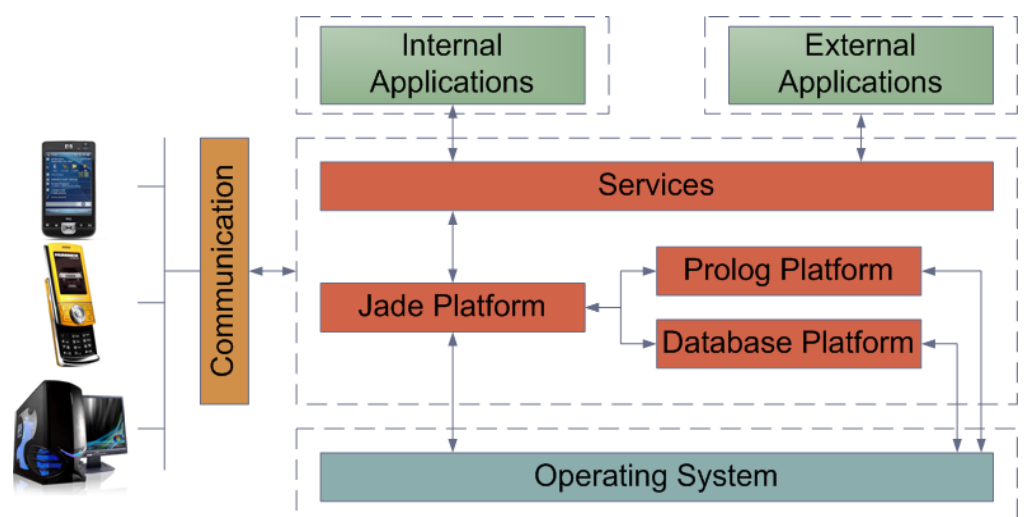

Fig. $\overline{2}$. Scheme of the Stages.

Due to its small size the messages are lightweight and easy to be transmitted between agents using the JADE platform message buffer, where all messages are saved even if the receiver agent is not active. A table and system of capturing and interpreting errors was created in order to the errors occurring during the processing phase can be relayed.

\section{Agenda Manager}

The Agenda Manager (AM) sets the bridge between the remaining parts of the manager system and the scheduling one, using the communication infrastructure available to receive and send requests. As result the AM stands for the communication and security of the whole systems. It configures a two stage application agent: it manages the incoming events to be scheduled and programs the time that triggers the Free Time Manager and it is also the communication input and output to the Client agents.

The AM supports the reception of multiple messages, and when it doesn't constitute a conflict in term of accessing the same calendar, the execution of the remaining agents at the same time, thus increasing the overall performance of the system. The AM ensures also that the user's friends and relatives can keep in touch with him and know what activities he is or will be doing.

Indeed, the AM manages the entire project. Its assessment modifies the way the project works.

\section{Conflicts Manager}

The Conflicts Manager (CM) agent is intended to assure that there is no overlap of activities and events. This module schedules or reorganizes the events that are relayed from the Agenda Manager, insuring that they are in accordance with the rest of the events. When a collision of different events is detected, the outcome 
will be decided by methods of intelligent conflict management. In case of overlapping events with the same priority level, the notification of overlapping is reported to the sender, so he/she may try to reschedule to a different time slot.

The events follow a hierarchy system. Every event has a value that is defining of his priority or urgency. Most of the conflicts will use the priorities value to be solved. This agent has also the capacity to manage all the connections with the other users as well as with the user relatives.

The Conflicts Manager operation can be explained in the following way:

1. When an operation is done by an administrator, the AM receives the message and calls the CM.

2. The CM enters in action by reading all the calendar files, parsing the new event and using the CLP engine to compare the priority levels of eventually conflicting events.

3. It is created a new ICS calendar ready to be delivered to the user.

4. A new message is then sent to the user and to the administrator, notifying them that a new Calendar is available and that the new insertion was successful.

\section{Free Time Manager}

The Free Time Manager (FTM) will schedule recreational activities in the free spaces of the user's calendar, in order to enforce the well being of the user. These activities configure an important milestone for an active ageing on the part of the user, once it promotes cultural, educational and conviviality conducts, based on an individualized plan. The FTM has a database that contains information of the user's favourite activities, previously verified by the decision support group or a medical committee. This agent supports the reception of a time trigger in order to be activated, always sent by the AM. It also as a built-in database connection in order to be able retrieve the available events and the logic engine responsible for all the decisions and changes made to the calendar.

The FTM uses a distribution function (2) defined attending some variables allowing it to decide the activity that is inserted into the user free time. For instance, in a three activities packet, the rate for the activity, from higher to lower priority, is $70 \%, 25 \%$ and $5 \%$. The activities are merely suggestive, it comes to the user to decide to execute them or not. On the other hand the activities chosen are those that fit into the time space that is available. 


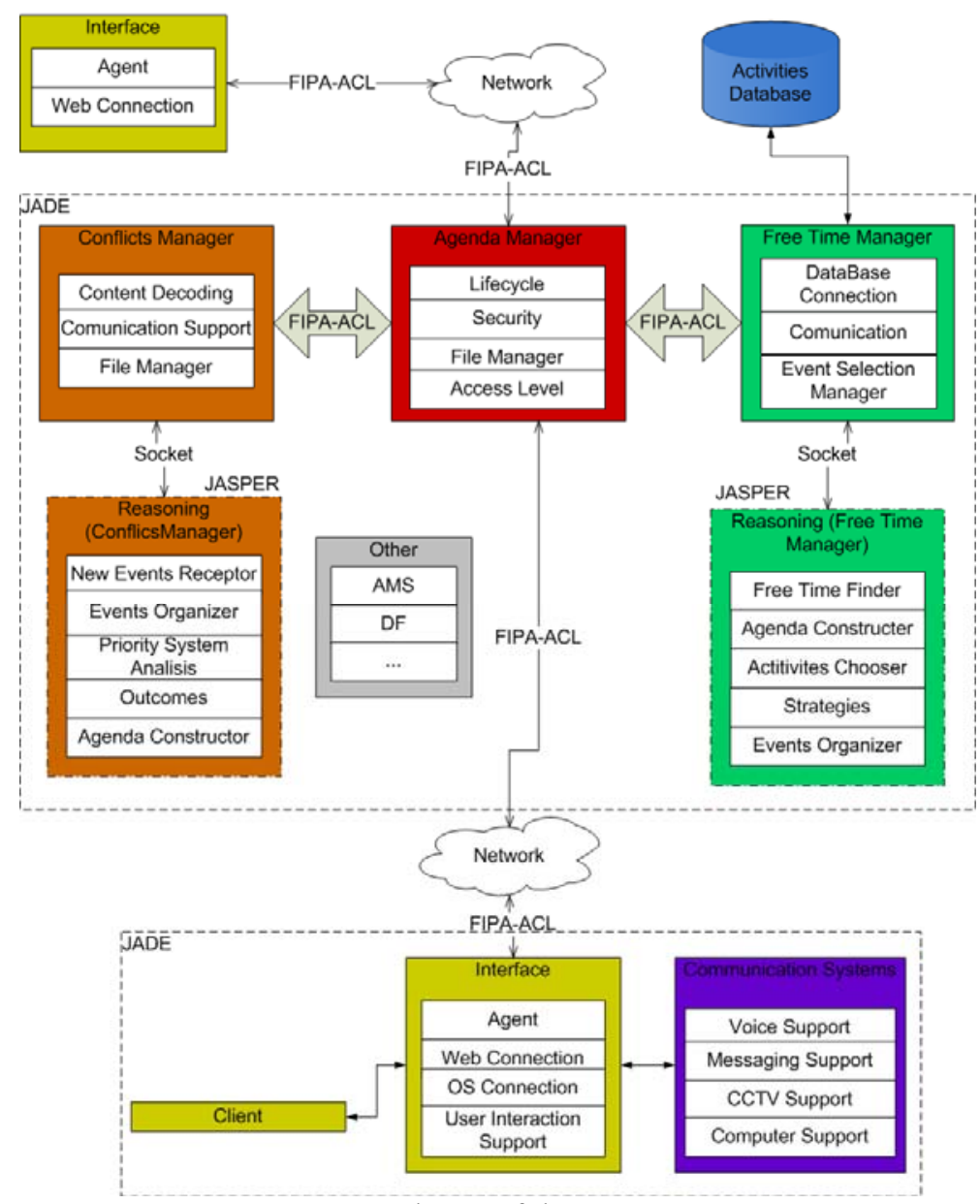

Fig. 3. Scheme of the Structure.

As the project evolved, especially during the investigation phase, we found other related projects oriented to elderly people like TimeBank [9] and ePal. Although these projects are not directly associated with the PMA area, their objective of keeping an interesting life through activities seemed to be a rather interesting way to the elderly. We believe these projects can be used by the FTM as activities supplier.

\section{Interface Manager}

The interface intends to be intuitive and easy to use. It is known that the elderly have some difficulties with new technologies, so the interface must be intuitive and easy to use. Large buttons are used and only the necessary information is 
displayed. A variable warning system is also available. When an event is triggered or accomplished, the user is informed.

This agent assures that the information reaches the user, by computer or mobile phone, so the user is always connected to the system. The information warnings and content can be textual, in audio format (pre-recorded messages) or both.

In terms of functionalities it supports the communication between the iGenda and supports also the distribution of the information through the already mentioned devices.

\section{Conclusions}

Currently most of the project bare bones are already working and we are currently focused in the remaining functionalities. Regardless of how it will evolve in the future, there are still problems and critical decisions to be made, namely the "density" problem, where by density we mean overcrowding the calendar of the user with too many activities, making it more stressful than relaxing. It also makes the difference to other PMAs, once it introduces the component of free time occupation, a problem to be addressed in terms of socialization; i.e., in terms of a process by which the user learn acceptable and unacceptable behaviours for a give environment. Also a Case Based Reasoning (CBR) system [10] [11] is under assessment to introduce the capability to record all of the user choices and actions to improve the future recommendations and actions taken as also to keep track as a log for future components improvement.

The mobile phone functionality is still under development but should be fully functional in the near future.

\section{References}

1. UNFPA: Population Ageing and Development: Operational Challenges In Developing Countries. (2002)

2. Tucker, G.: Age-Associated Memory Loss: Prevalence and Implications. Journal Watch Psychiatry (1995)

3. Aguilar, J.-M., Cantos, J., Expósito, G., Gómez, P.: Tele-assistance Services to Improve the Quality of Life for Elderly Patients and their Relatives: The Tele-CARE Approach. The Journal on Information Technology in Healthcare (2004)

4. Brown, S.: Next generation telecare and its role in primary and community care. Health \& Social Care in the Community 11, 459-462 (2003)

5. Costa, Â., Novais, P., Costa, R., Neves, J.: Memory Assistant In Everyday Living. ESM'2009 - The 23rd annual European Simulation and Modelling Conference (2009)

6. Fraile, J., Tapia, D., Rodríguez, S., Corchado, J.: Agents in Home Care: A Case Study. In: Hybrid Artificial Intelligence Systems 5572/2009. Springer Berlin / Heidelberg (2009)

7. Luck, M., Ashri, R., d'Inverno, M.: Agent-Based Software Development. Artech House Publishers (2004)

8. Moreno, A., Valls, A., Viejo, A.: Using JADE-LEAP to implement agents in mobile devices. In: http://jade.tilab.com/papers/EXP/02Moreno.pdf. 
9. Pokahr, A., Braubach, L.: From a Research to an Industrial-Strength Agent Platform: Jadex V2. 9. Internationale Tagung Wirtschaftsinformatik (2009)

10. Castolo, O., Ferrada, F., Camarinha-Matos, L.: TeleCARE Time Bank: A Virtual Community for Elderly Care Supported by Mobile Agents. The Journal on Information Technology in Healthcare, 119-133 (2004)

11. Corchado, J., Bajo, J., Abraham, A.: GERAmI: Improving the delivery of health care. In: IEEE Intelligent Systems. Special Issue on Ambient Intelligence 2nd edn. 23. (2008) 19-25

12. Corchado, J., Bajo, J., Paz, Y., Tapia, D.: Intelligent Environment for Monitoring Alzheimer Patients, Agent Technology for Health Care. In : Decision Support Systems 2nd edn. 44. (2008) 382-396 